\title{
Quantifying type I and type II errors in decision-making under uncertainty: the case of GM crops
}

\author{
Erik Ansink · Justus Wesseler
}

Received: 4 December 2008 / Accepted: 20 February 2009 / Published online: 28 February 2009

(C) The Author(s) 2009. This article is published with open access at Springerlink.com

\begin{abstract}
In a recent paper, Hennessy and Moschini (American Journal of Agricultural Economics 88(2): 308-323, 2006) analyse the interactions between scientific uncertainty and costly regulatory actions. We use their model to analyse the costs of making type I and type II errors, in the context of the possible introduction of GM crops. We demonstrate that the costs of making a type I or type II error should be calculated as the difference in costs between choosing the right action immediately and choosing the right action only after the state of nature has been revealed. The importance of this result for the discussion on the precautionary principle is briefly examined.
\end{abstract}

Keywords Type I and type II errors · Uncertainty · Irreversibility · GM crops

JEL Classification D81 - Q16

\section{Introduction}

The introduction of genetically modified (GM) crops has raised concerns about the implications for biodiversity and ecosystems in many countries (Hails 2002; Ervin and Welsh 2005). Once GM crops are introduced, gene flow and non-target effects may yield irreversible costs to society. One of the objectives of regulatory bodies such as the European Food Safety Authority (EFSA) is to assess the likelihood of such a scenario. To analyse the trade-off between (irreversible) costs and benefits

\footnotetext{
E. Ansink (凶) · J. Wesseler

Environmental Economics and Natural Resources Group, Wageningen University, P.O. Box 8130, 6700 EW Wageningen, The Netherlands

e-mail: erikansink@gmail.com

J. Wesseler

e-mail: justus.wesseler@wur.nl
} 
of a regulation on GM crops, both uncertainty and irreversibility have to be explicitly taken into account. Under uncertainty and irreversibility, the costs of making an initially incorrect decision may be high. Such an incorrect decision can be prevented by delaying the decision until additional information has arrived. This delay, however, comes at a cost too.

In a recent article in the American Journal of Agricultural Economics, Hennessy and Moschini (2006) (from here on referred to as HM) analyse the interactions between scientific uncertainty and costly regulatory actions. The setting of their article is a regulator having to decide whether to ban a certain practice or technology when there is uncertainty on its possible damage (for instance GM crops). Their main results are: the presence of adjustments costs "creates inertia and favours the status quo" and adjustment costs "change how the optimal regulation responds to better scientific information". Their paper makes an important theoretical contribution to the assessment of additional information to decision-making about regulatory policies. While the importance of new information is their major contribution, HM also suggest that the specification of their model allows to quantify the type I and type II errors within the decision-making process. The objective of this paper is to provide a specification of type I and type II errors in the HM model and discuss its importance.

\section{Model}

HM set up a model where a regulator faces the choice between banning and not banning at time $t=0$. With probability $q$, the practice causes damage and with probability $1-q$ it does not. $\kappa \in(0, \infty)$ is the date at which uncertainty is resolved; it follows from the exponential distribution $f(\kappa)=h e^{-h \kappa}$, with $E(\kappa)=1 / h$, where $h$ is the hazard rate. ${ }^{1}$ There are three types of costs related to the implementation of a ban: adjustment costs $\delta_{1}$ are the costs of implementing the ban, adjustment costs $\delta_{2}$ are the costs of reversing the ban (if necessary), and $C$ is an annual cost that lasts as long as the ban is in place. $R(a, s, \kappa)$ denotes the realized total costs to the regulator, where $a_{t} \in\{B, N\}$ is the action chosen at time $t$ (Ban or No ban), and $s \in\{T, F\}$ is the state of nature (True or False that the practice causes damage). When the ban is in place and the practice indeed causes damage, annual damage $D$ is avoided. All costs and benefits are discounted using the continuous-time discount rate $r$.

Implicit in $\mathrm{HM}$ is the definition of the $H_{0}$ and $H_{1}$ hypotheses:

$\mathrm{H}_{0}$ : The state of nature is $T$; the regulator should ban (given the correct assumption by HM that $\left.D>C+\delta_{1} r\right)$;

$\mathrm{H}_{1}$ : The state of nature is $F$; the regulator should not ban.

Notice that this specification of the $H_{0}$ and $H_{1}$ hypotheses is somewhat counterintuitive. The conventional assumption is that $H_{0}$ represents the state of nature without damage. For completeness, recall the definitions of type I and type II errors:

\footnotetext{
${ }^{1}$ The exponential distribution has a couple of attractive features for models with arrival of information; an important feature is that it allows analytical tractability of the model.
} 
Type I error: The error of rejecting the null hypothesis when it is actually true; i.e. not banning when the regulator should have;

Type II error: The error of accepting the null-hypothesis when it is actually false; i.e. banning when the regulator should not have.

HM calculate the costs of a type I error $\left(Z_{H M}^{I}\right)$ as the ex ante expected total costs of not banning, and the costs of a type II error $\left(Z_{H M}^{I I}\right)$ as the ex ante expected total costs of banning. This is most easily illustrated using their baseline model without intermediate arrival of information, while the same holds for their three period-model with gradual resolution of uncertainty:

$$
\begin{aligned}
Z_{H M}^{I}= & E[q R(N, T, \kappa)+(1-q) R(N, F, \kappa)] \\
= & \int_{0}^{\infty}[q R(N, T, \kappa)+(1-q) R(N, F, \kappa)] f(\kappa) d \kappa \\
= & \int_{0}^{\infty}\left[q\left(\delta_{1} e^{-r \kappa}+\int_{\kappa}^{\infty} C e^{-r t} d t+\int_{0}^{\kappa} D e^{-r t} d t\right)\right] f(\kappa) d \kappa \\
= & \frac{(q / r)\left(\delta_{1} h r+C h+D r\right)}{r+h} ; \\
Z_{H M}^{I I}= & E[q R(B, T, \kappa)+(1-q) R(B, F, \kappa)] \\
= & \int_{0}^{\infty}[q R(B, T, \kappa)+(1-q) R(B, F, \kappa)] f(\kappa) d \kappa \\
= & \int_{0}^{\infty}\left[q\left(\delta_{1}+\int_{0}^{\infty} C e^{-r t} d t\right)\right. \\
& \left.+(1-q)\left(\delta_{1}+\delta_{2} e^{-r \kappa}+\int_{0}^{\kappa} C e^{-r t} d t\right)\right] f(\kappa) d \kappa \\
= & \frac{C+\delta_{1} r+\left(\delta_{1}+\delta_{2}\right) h}{r+h}+q\left[\frac{C h-\delta_{2} h r}{r(r+h)}\right] ;
\end{aligned}
$$

where $E$ is the expectation operator.

This specification of the costs of making type I and type II errors does not consider the benefits of arrival of new information. We choose to explicitly consider these benefits; thereby following the literature on decision-making under uncertainty and irreversibility to which HM contribute (cf. Dixit and Pindyck 1994). Given the definitions of the $\mathrm{H}_{0}$ and $\mathrm{H}_{1}$ hypotheses introduced above, we therefore propose to calculate the costs of making a type I or type II error as the difference in costs between choosing the right action immediately and choosing the right action only after the state of nature has been revealed. This implies that the cost of making a type I error $\left(Z_{A W}^{I}\right)$ is the difference in costs between immediate banning $\left(a_{0}=a_{\kappa}=B\right)$ and delayed banning ( $a_{0}=N$ and $a_{\kappa}=B$ ), given that the state of nature is $T$. Similarly, the cost of making a type II error $\left(Z_{A W}^{I I}\right)$ is the difference in costs between immediate not banning $\left(a_{0}=a_{\kappa}=N\right)$ and delayed not banning $\left(a_{0}=B\right.$ and $\left.a_{\kappa}=N\right)$, given that the state of nature is $F$ : 


$$
\begin{aligned}
Z_{A W}^{I}= & E[R(N, T, \kappa)-R(B, T, \kappa)] \\
= & \int_{0}^{\infty}[R(N, T, \kappa)-R(B, T, \kappa)] f(\kappa) d \kappa \\
= & \int_{0}^{\infty}\left[\left(\delta_{1} e^{-r \kappa}+\int_{\kappa}^{\infty} C e^{-r t} d t+\int_{0}^{\kappa} D e^{-r t} d t\right)\right. \\
& \left.-\left(\delta_{1}+\int_{0}^{\infty} C e^{-r t} d t\right)\right] f(\kappa) d \kappa \\
= & \frac{D-C-\delta_{1} r}{r+h} ; \\
Z_{A W}^{I I}= & E[R(B, F, \kappa)-R(N, F, \kappa)] \\
= & \int_{0}^{\infty}[R(B, F, \kappa)-R(N, F, \kappa)] f(\kappa) d \kappa \\
= & \int_{0}^{\infty}\left[\left(\delta_{1}+\delta_{2} e^{-r \kappa}+\int_{0}^{\kappa} C e^{-r t} d t\right)-0\right] f(\kappa) d \kappa \\
= & \frac{C+\delta_{1} r+\left(\delta_{1}+\delta_{2}\right) h}{r+h} .
\end{aligned}
$$

Obviously, because we use the HM model to construct our specification, we depend on their distributional assumption for $\kappa$, see footnote 1 .

Comparing the size of errors calculated by HM and by us (AW) we derive that:

$$
\begin{aligned}
& Z_{H M}^{I} \gtrless Z_{A W}^{I} \quad \Leftrightarrow \quad q \gtrless \frac{D r-C r-\delta_{1} r^{2}}{D r+C h+\delta_{1} h r} \\
& Z_{H M}^{I I} \gtrless Z_{A W}^{I I} \quad \Leftrightarrow \quad C \gtrless \delta_{2} r
\end{aligned}
$$

Compared with the AW specification, the HM specification results in higher costs of making a type I error if $q$ is large and vice versa. For type II errors, the HM specification results in higher costs as long as $C>\delta_{2} r$, and the difference is larger when $q$ is higher. It is reasonable to assume that $C>\delta_{2} r$, because this assures that, when $a_{0}=B$ and $s=F$, the adjustment $\operatorname{costs} \delta_{2}$ do not prevent the adjustment to $a_{\kappa}=N$. Compared with the AW specification, for the relevant parameter values, the HM specification results in higher costs of making a type II error. The AW specification is therefore more conservative, and more so for larger values of $q$.

An important difference between the two specifications is that the AW specification of type I and type II errors is independent of $q$, the probability that damage occurs. $^{2}$ This makes sense, as the costs and the probability of making an error are two distinct notions. When $q$ increases, this should not affect the costs of an incorrect decision. It should, however, affect the expected costs of making type I and type II errors, calculated as costs times probability. Hence, the HM specification is unnecessarily sensitive to $q$.

\footnotetext{
${ }^{2}$ Thanks to an anonymous reviewer who pointed out this difference.
} 


\section{Discussion}

The HM specification results in higher costs of not banning when the regulator should have (type I error), if $q$, the probability that damage occurs, is large, and vice versa. For banning when the regulator should not have (type II error), the HM specification always results in higher costs for relevant parameter values.

To our knowledge this is the first attempt at specifying the costs of making type I or type II errors in a model of decision-making under uncertainty and irreversibility. A topic of utmost importance for decision-making with respect to the introduction of GM crops (Wesseler et al. 2007). The importance of appropriately specifying errors in this type of models is apparent for two reasons. First, from an economic point of view, it is important to know the costs and probabilities of making type I and type II errors in order to calculate the expected costs of making an error. ${ }^{3}$ A regulator may introduce a threshold value for the expected costs of making a type I or type II error in his decision-making. Within the debate about bio-safety and food safety, the economic specification allows to compare the costs of additional tests with the changes in costs of making either the type I or type II error.

Second, it is important as an input for the application of the precautionary principle (cf. Gollier and Treich 2003). The precautionary principle has gained increasing importance in policy-making; it has for instance been adopted by the EU on the issue of releasing GM crops. One interpretation of the precautionary principle is the reversing of the burden of proof (van den Belt 2003). In the context of this model this interpretation implies that the probability of making a type I error should be minimized (Ervin and Welsh 2005). ${ }^{4}$ Clearly, a proper understanding of the specification of the costs of type I errors is a prerequisite for a sound use (or disuse) of the precautionary principle.

Acknowledgements We like to thank two anonymous reviewers for helpful suggestions.

Open Access This article is distributed under the terms of the Creative Commons Attribution Noncommercial License which permits any noncommercial use, distribution, and reproduction in any medium, provided the original author(s) and source are credited.

\section{References}

Dixit, A.K., Pindyck, R.S.: Investment under Uncertainty. Princeton University Press, Princeton (1994)

Ervin, D.E., Welsh, R.: Environmental effects of genetically modified crops: differentiated risk assessment and management. In: Wesseler, J.H.H. (ed.) Environmental Costs and Benefits of Transgenic Crops. Springer, Dordrecht (2005)

Gollier, C., Treich, N.: Decision-making under scientific uncertainty: the economics of the precautionary principle. J. Risk Uncertain. 27(1), 77-103 (2003)

\footnotetext{
${ }^{3}$ Note that the probability of making type I and type II errors depends on uncertainty and the selected significance level, where the significance level reflects the relative importance attached to type I and type II errors.

${ }^{4}$ Due to the specification of the $\mathrm{H}_{0}$ and $\mathrm{H}_{1}$ hypotheses in this model, the precautionary principle asks for minimization of type I errors instead of the conventional minimization of type II errors.
} 
Hails, R.S.: Assessing the risks associated with new agricultural practices. Nature 418(6898), 685-688 (2002)

Hennessy, D.A., Moschini, G.: Regulatory actions under adjustment costs and the resolution of scientific uncertainty. Am. J. Agric. Econ. 88(2), 308-323 (2006)

van den Belt, H.: Debating the precautionary principle: guilty until proven innocent or innocent until proven guilty? Plant Physiol. 132(3), 1122-1126 (2003)

Wesseler, J., Scatasta, S., Nillesen, E.: The maximum incremental social tolerable irreversible costs (MISTICs) and other benefits and costs of introducing transgenic maize in the EU-15. Pedobiologia 51(3), 261-269 (2007) 